\title{
Creencias y percepciones de los pacientes con estreñimiento crónico sobre etiología, complicaciones y eficacia de las medidas generales. Una encuesta en consulta externa de gastroenterología
}

\section{Outpatient gastroenterology survey of the beliefs and perceptions of patients with chronic constipation regarding etiology, complications and efficacy of general measures}

Adrián Díaz Mogollón, MD, ${ }^{1}$ William Otero Regino, MD, ${ }^{2^{\star}}$ Lina Otero Parra, MD. ${ }^{3}$

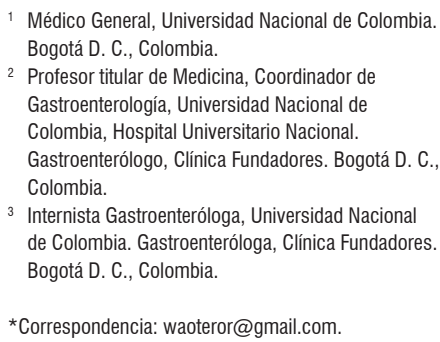

2 Profesor titular de Medicina, Coordinador de Gastroenterología, Universidad Nacional de Colombia, Hospital Universitario Nacional. Gastroenterólogo, Clínica Fundadores. Bogotá D. C. Colombia.

3 Internista Gastroenteróloga, Universidad Nacional de Colombia. Gastroenteróloga, Clínica Fundadores. Bogotá D. C., Colombia.

*Correspondencia: waoteror@gmail.com.

\begin{abstract}
Resumen
Introducción: el estreñimiento crónico (EC) afecta al 14 \% de la población mundial y genera altos costos para los sistemas de salud por los diferentes tratamientos. Con frecuencia, pacientes y médicos tienen percepciones erróneas sobre la etiología, complicaciones y el tratamiento de la entidad con medidas generales. Materiales y métodos: se realizó una encuesta a pacientes con EC atendidos en la consulta externa de un servicio de gastroenterología de una institución universitaria. Con interrogantes todos falsos, se investigaron las creencias erróneas más frecuentes en los pacientes con diagnóstico de estreñimiento respecto a su enfermedad. Resultados: se encuestaron 278 pacientes que cumplieron con los criterios de inclusión. El $98 \%$ consideró que el EC es la causa de las hemorroides, mismo que produce intoxicación (86 \%) y causa cáncer de colon (78 \%). Otros conceptos erróneos fueron el bajo consumo de fibra (79 \%) como causa de la enfermedad, al igual que la baja actividad física $(52 \%)$. No consideran útil para el tratamiento la dieta alta en fibra (55\%) ni realizar ejercicio de forma regular (69\%). La observación más frecuente fue el consumo abundante de fibra sin presentar mejoría.
\end{abstract}

Palabras clave

Encuesta, estreñimiento, percepciones, fibra, cáncer, hemorroides, ejercicio.

\begin{abstract}
Introduction: Chronic constipation affects $14 \%$ of the world's population. It leads to high costs for health systems due to diverse treatments. Frequently, patients and doctors have erroneous perceptions about constipation's etiology, complications and treatment. Materials and methods: Patients with chronic constipation were surveyed at the outpatient clinic of a gastroenterology service of a university institution. The most frequent erroneous beliefs regarding the condition found among patients diagnosed with constipation were investigated. Results: A total of 278 patients who met the inclusion criteria were surveyed. Ninety-eight percent consider that chronic constipation is the cause of hemorrhoids, eighty-six percent believe that it leads to poisoning, and seventy-eight percent believe that it causes colon cancer. Other misconceptions were that low fiber consumption causes constipation (79\%) and that low levels of physical activity cause constipation (52\%). Fifty-five percent do not consider that high fiber diets (55\%) or regular exercise $(69 \%)$ are useful for treatment. The most frequent observation was abundant consumption of fiber without improvement.
\end{abstract}

Keywords

Survey, constipation, perceptions, fiber, cancer, hemorrhoids, exercise. 


\section{INTRODUCCIÓN}

El estreñimiento crónico (EC) afecta globalmente al $14 \%$ de los pacientes adultos y al $30 \%$ de los que tienen más de 60 años $(1,2)$; es más frecuente en mujeres y en personas institucionalizadas $(1,2)$; se asocia con un estado socioeconómico bajo y con enfermedades psiquiátricas $(1,3)$; altera notablemente la calidad de vida (4); y, adicionalmente, por su alta prevalencia, es costoso para los sistemas de salud $(3,4)$. En estados Unidos (EE. UU.) genera 8 millones de consultas ambulatorias anuales y un costo superior a 230 millones de dólares (5); además, 1,2 millones de pacientes son remitidos a gastroenterología por dicha patología (4, $5)$. En ese país se ha encontrado que los costos médicos directos anuales en la población femenina son casi el doble que la de las mujeres sin estreñimiento $(2,5)$. En EE. UU. hay 2,5 millones de personas con EC, que si son sometidas a colonoscopias no indicadas, el costo de la realización de estas alcanzaría los 5 mil millones de dólares anuales $(5,6)$.

Con respecto a su causa y tratamiento, existen muchas creencias populares y mitos que se han perpetuado no solo en los pacientes, sino también en muchos médicos (7). Entre esos, los más frecuentes son los siguientes: que el bajo consumo de fibra es una de sus causas, es inductor de cáncer de colon (CC; produce autointoxicación, se trata ingiriendo grandes cantidades de agua) $(7,8)$. Otras creencias sobre el tratamiento es que se alivia con el consumo de probióticos, solos o con yogurt; y, así mismo, que el ejercicio produce mejoría del mismo.

Teniendo en cuenta su alta prevalencia, los altos costos identificados en otros países y la alta frecuencia percibida (no medida) en la consulta externa de gastroenterología, decidimos realizar el presente trabajo en la unidad de gastroenterología y endoscopia digestiva de la Clínica Fundadores de Bogotá, una institución de tercer nivel, adscrita al posgrado de gastroenterología de la Universidad Nacional de Colombia.

\section{MATERIALES Y MÉTODOS}

Esta investigación se realizó en pacientes con diagnóstico de EC utilizando los criterios de ROMA IV (9), atendidos en la consulta externa de Gastroenterología de la Clínica Fundadores, de Bogotá D. C., Colombia. Se incluyeron pacientes que consultaron entre mayo y octubre de 2016, quienes después de ser instruidos sobre la naturaleza y propósitos de la investigación, firmaron el consentimiento y voluntariamente decidieron participar en el estudio. La información se recolectó con base en un instrumento diseñado específicamente para esta investigación, que incluía datos demográficos y 14 preguntas dicotómicas basadas en las inquietudes y dudas identificadas que expresaban los pacientes en la consulta de gastroenterología. Esas inquietudes se identificaron durante los 6 meses previos al inicio de la presente investigación. En el formulario adicionalmente se dejó un espacio abierto donde los pacientes encuestados podían escribir cualquier comentario o creencia adicional sobre la enfermedad. El formulario fue aplicado por uno de nosotros $(\mathrm{ADM})$, durante su pasantía especial de internado en la unidad de gastroenterología. Los pacientes atendidos pertenecen a estratos socioeconómicos 2,3 y 4 .

\section{RESULTADOS}

Se incluyeron en el estudio a 278 pacientes, de los cuales el $75 \%$ era de sexo femenino; la edad promedio era de 50 años; todos habían recibido tratamiento inicial con fibra, específicamente, salvado de trigo y psyllium (30 gramos), y remedios caseros. Con esas medidas, el $20 \%$ de los pacientes había mejorado. El 80 \% restante, al momento de la encuesta recibía polietilenglicol 3350 (17 g/día) (Contumax, PEG $^{*}$ ) o picosulfato de sodio 5-10 mg al día. Estos pacientes referían estar satisfechos con estos dos últimos tipos de tratamiento por la importante mejoría de sus síntomas. Las preguntas y las frecuencias de las mismas, que son el objetivo del presente trabajo, se muestran en la Figura 1.

\section{DISCUSIÓN}

Los resultados del presente estudio muestran que los pacientes de esta población colombiana también tienen muchas de las creencias encontradas en estudios similares de otros países $(7,8)$. Las más frecuentes fueron que el EC es la causa de las hemorroides (98\%), causa intoxicación $(86 \%)$ y también que causa CC. Con respecto a esta última, no hay ninguna relación ya que el CC está asociado fundamentalmente con historia familiar de CC, tabaquismo, consumo del alcohol, obesidad, diabetes, alto consumo de carnes procesadas y enfermedad inflamatoria intestinal (10). De manera similar, no hay evidencia definitiva de que el estreñimiento produzca hemorroides (7).

Llama la atención que, aunque la mayoría de los pacientes $(79 \%)$ considera como causa de estreñimiento la falta de fibra en la dieta, más de la mitad (55\%) no cree que una forma de fibra como las frutas tenga utilidad en el tratamiento. Esta última apreciación probablemente es el resultado de no haber mejorado con la utilización empírica de altas cantidades de la misma. En los trabajos de investigación al respecto no se ha demostrado un claro beneficio de la fibra (11). En un metaanálisis reciente se encontró un beneficio marginal con el psyllium; sin embargo, los trabajos tenidos en cuenta en esa investigación tenían múltiples sesgos metodológicos y eran de pobre calidad (12). Aunque algunas guías de manejo actuales recomiendan 


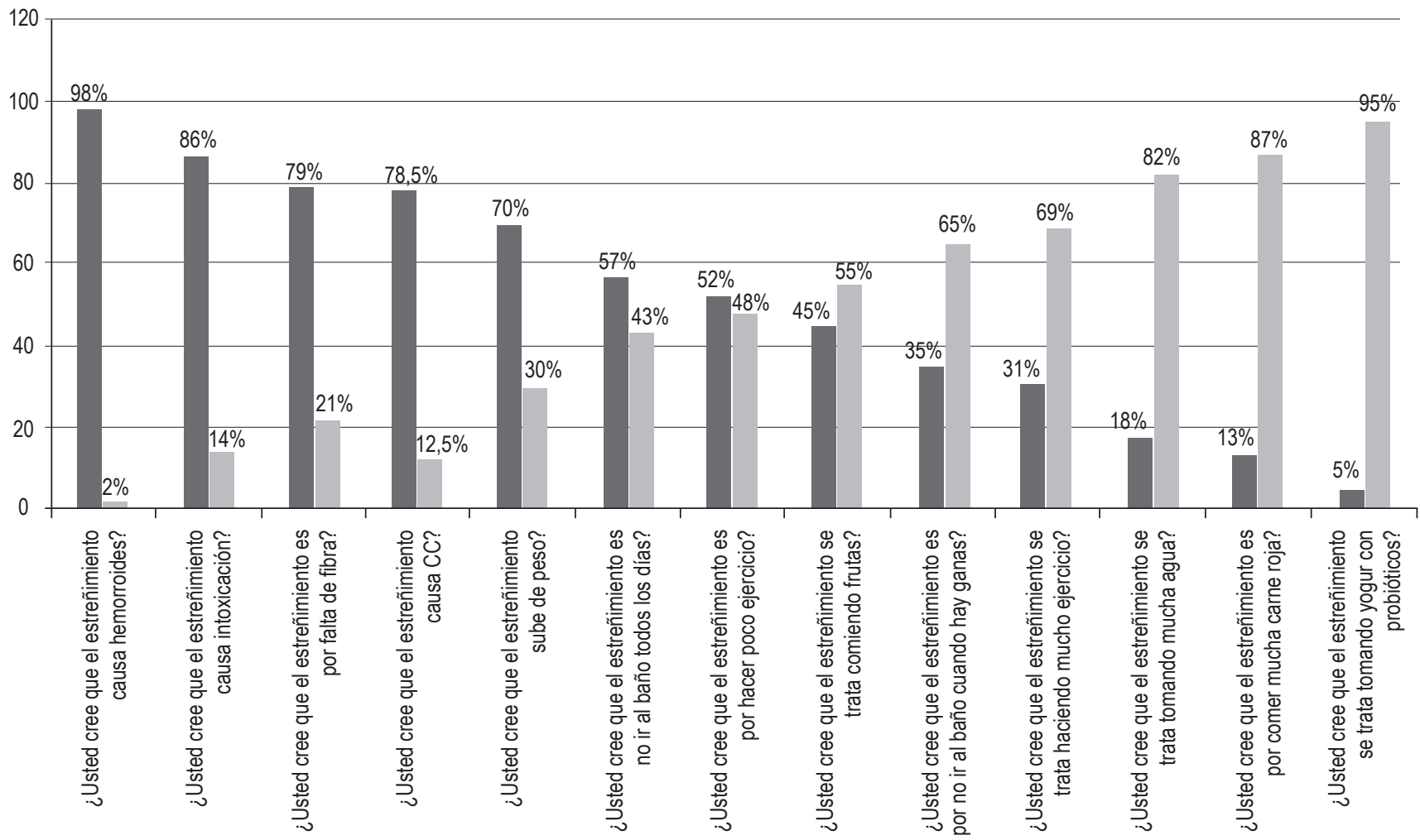

Figura 1. Preguntas y respuestas de los pacientes encuestados.

aumentar el consumo de fibra en el tratamiento inicial (1315), la evidencia que respalda esas recomendaciones es de baja calidad $(11,12)$. Adicionalmente, la fibra insoluble puede inducir o exacerbar la flatulencia, y en algunos puede empeorar el estreñimiento $(12,16)$. La fibra soluble como el psyllium o ispaghula ha mostrado un beneficio marginal; sin embargo, a largo plazo, los resultados no se mantienen (13-15). No obstante la evidencia publicada, la mayoría de los médicos sigue considerando que la fibra y las frutas son fundamentales en el manejo del EC.

El $52 \%$ de los encuestados considera que la falta de ejercicio es un factor etiológico del estreñimiento; sin embargo, paradójicamente el $69 \%$ no cree que realizar ejercicio sirva como tratamiento. Los estudios realizados al respecto no han demostrado que hacer ejercicio de manera regular mejore el estreñimiento o disminuya la necesidad de laxantes $(17,18)$. Una inferencia cotidiana del sentido común es que, si el ejercicio aumentara el tránsito del colon y favoreciera las deposiciones, entonces los deportistas de alto rendimiento no podrían competir porque con frecuencia tendrían diarrea. El ejercicio tiene efectos benéficos demostrados para el aparato cardiovascular (19).

En relación con el beneficio de consumir mucho líquido para tratar el estreñimiento, el 82 \% respondió que no consideraba que fuera útil. Esa apreciación es cierta ya que no hay evidencia que demuestre el beneficio de tomar mucho líquido sobre esta entidad $(11,20)$, excepto en los pacientes hospitalizados y deshidratados (11). Con respecto a los probióticos, se ha encontrado que pueden aumentar el transito colónico, pero no se ha demostrado que alivien el estreñimiento ni en adultos $(21,22)$ ni en niños $(23)$. La mayoría de los encuestados ( $85 \%$ ) no consideran que el yogur con probióticos sea útil en la entidad.

Contrario a las creencias populares, el manejo del EC es complejo debido a la heterogeneidad de los pacientes, los cuales se pueden clasificar en los siguientes subgrupos (3, 9, 24, 25): pacientes con tránsito colónico normal, tránsito lento, disfunción del piso pélvico (anismo, disinergia del 
piso pélvico) y estreñimiento secundario a opioides. Este último hace parte del síndrome de intestino narcótico, una entidad con gran morbimortalidad (26).

Las estrategias farmacológicas con eficacia demostrada incluyen fundamentalmente laxantes osmóticos (polietilenglicol, lactulosa), laxantes estimulantes (picosulfato de sodio), secretagogos (linaclotida, lubiprostona), prucaloprida (un procinético universal), agonista 5HT4 (27) y metilnaltrexona y alvimopan para el estreñimiento secundario a opioides (28).

\section{CONCLUSIONES}

La mayoría de los pacientes cree erróneamente que el estreñimiento causa CC y hemorroides. La gran mayoría no considera de utilidad la ingesta de frutas para tratar el estreñimiento. Los pacientes consideran que el ejercicio no sirve para tratar esta enfermedad. Acorde con los resultados y la evidencia científica, la apreciación más frecuente dada por los pacientes es acertada, ya que aunque atribuyan como factores etiológicos el poco consumo de fibra y la falta de ejercicio, la mayoría no consideró finalmente que el aumento en los mismos mejore el estreñimiento. Es posible que las respuestas acertadas de la población estudiada, al respecto de las creencias populares, se deba a que muchos de ellos asisten a las charlas educativas que un miembro de nuestro servicio (WO) les brinda sobre patologías crónicas.

\section{Conflictos de intereses}

Ninguno.

\section{REFERENCIAS}

1. Suares NC, Ford AC. Prevalence of, and risk factors for, chronic idiopathic constipation in the community: systematic review and meta-analysis. Am J Gastroenterol. 2011;106(9):158291; quiz 1581, 1592. doi: 10.1038/ajg.2011.164.

2. Mugie SM, Benninga MA, Di Lorenzo C. Epidemiology of constipation in children and adults: a systematic review. Best Pract Res Clin Gastroenterol. 2011;25(1):3-18. doi: 10.1016/j.bpg.2010.12.010.

3. Camilleri M, Ford AC, Mawe GM, Dinning PG, Rao SS, Chey WD, et al. Chronic constipation. Nat Rev Dis Primers. 2017;3:17095. doi: 10.1038/nrdp.2017.95.

4. Dennison C, Prasad M, Lloyd A, Bhattacharyya SK, Dhawan R, Coyne $\mathrm{K}$. The health-related quality of life and economic burden of constipation. Pharmacoeconomics. 2005;23(5):461-76. doi: 10.2165/00019053-200523050-00006.

5. Choung RS, Branda ME, Chitkara D, Shah ND, Katusic SK, Locke GR 3rd, et al. Longitudinal direct medical costs associated with constipation in women. Aliment Pharmacol Ther. 2011;33(2):251-60. doi: 10.1111/j.1365-2036.2010.04513.x.
6. Martin BC, Barghout V, Cerulli A. Direct medical costs of constipation in the United States. Manag Care Interface. 2006; 19(12):43-9.

7. Müller-Lissner SA, Kamm MA, Scarpignato C, Wald A. Myths and misconceptions about chronic constipation. Am J Gastroenterol. 2005;100(1):232-42. doi: 10.1111/j.15720241.2005.40885.x.

8. Ziegenhagen DJ, Tewinkel G, Kruis W, Herrmann F. Adding more fluid to wheat bran has no significant effects on intestinal functions of healthy subjects. J Clin Gastroenterol. 1991;13(5):525-30.

9. Mearin F, Lacy BE, Chang L, Chey WD, Lembo AJ, Simren M, et al. Bowel Disorders. Gastroenterology. 2016. pii: S00165085(16)00222-5. doi: 10.1053/j.gastro.2016.02.031.

10. Kolligs FT. Diagnostics and Epidemiology of Colorectal Cancer. Visc Med. 2016;32(3):158-64. doi: 10.1159/000446488.

11. Fathallah N, Bouchard D, de Parades V. Diet and lifestyle rules in chronic constipation in adults: From fantasy to reality.... Presse Med. 2017;46(1):23-30. doi: 10.1016/j. lpm.2016.03.019.

12. Christodoulides S, Dimidi E, Fragkos KC, Farmer AD, Whelan K, Scott SM. Systematic review with meta-analysis: effect of fibre supplementation on chronic idiopathic constipation in adults. Aliment Pharmacol Ther. 2016;44(2):10316. doi: 10.1111 /apt.13662.

13. Ford AC, Moayyedi P, Lacy BE, Lembo AJ, Saito YA, Schiller LR, et al. American College of Gastroenterology monograph on the management of irritable bowel syndrome and chronic idiopathic constipation. Am J Gastroenterol. 2014;109 Suppl 1:S2-26; quiz S27. doi: 10.1038/ajg.2014.187.

14. TutejaAK, TalleyNJ,Joos SK, WoehlJV,Hickam DH. Is constipation associated with decreased physical activity in normally active subjects? Am J Gastroenterol. 2005;100(1):124-9. doi: $10.1111 /$ j.1572-0241.2005.40516.x.

15. Bharucha AE, Pemberton JH, Locke GR 3rd. American Gastroenterological Association technical review on constipation. Gastroenterology. 2013;144(1):218-38. doi: 10.1053/j.gastro.2012.10.028.

16. Markland AD, Palsson O, Goode PS, Burgio KL, BusbyWhitehead J, Whitehead WE. Association of low dietary intake of fiber and liquids with constipation: evidence from the National Health and Nutrition Examination Survey. Am J Gastroenterol. 2013;108(5):796-803. doi: 10.1038/ ajg.2013.73.

17. Eswaran S, Muir J, Chey WD. Fiber and functional gastrointestinal disorders. Am J Gastroenterol. 2013;108(5):71827. doi: 10.1038/ajg.2013.63.

18. Daley AJ, Grimmett C, Roberts L, Wilson S, Fatek M, Roalfe A, et al. The effects of exercise upon symptoms and quality of life in patients diagnosed with irritable bowel syndrome: a randomised controlled trial. Int J Sports Med. 2008;29(9):778-82. doi: 10.1055/s-2008-1038600.

19. Karjalainen JJ, Kiviniemi AM, Hautala AJ, Piira OP, Lepojärvi ES, Perkiömäki JS, et al. Effects of physical activity and exercise training on cardiovascular risk in coronary artery disease 
patients with and without type 2 diabetes. Diabetes Care. 2015;38(4):706-15. doi: 10.2337/dc14-2216.

20. Young RJ, Beerman LE, Vanderhoof JA. Increasing oral fluids in chronic constipation in children. Gastroenterol Nurs. 1998;21(4):156-61.

21. Quigley EM. The enteric microbiota in the pathogenesis and management of constipation. Best Pract Res Clin Gastroenterol. 2011;25(1):119-26. doi: 10.1016/j. bpg.2011.01.003.

22. Dimidi E, Christodoulides S, Scott SM, Whelan K. Mechanisms of Action of Probiotics and the Gastrointestinal Microbiota on Gut Motility and Constipation. Adv Nutr. 2017;8(3):484-494. doi: 10.3945/an.116.014407.

23. Wojtyniak K, Szajewska H. Systematic review: probiotics for functional constipation in children. Eur J Pediatr. 2017;176(9):1155-1162. doi: 10.1007/s00431-017-2972-2.
24. Serrano-Falcón B, Rey E. The safety of available treatments for chronicconstipation. ExpertOpinDrugSaf.2017;16(11):12431253. doi: 10.1080/14740338.2017.1361402.

25. Krogh K, Chiarioni G, Whitehead W. Management of chronic constipation in adults. United European Gastroenterol J. 2017;5(4):465-472. doi: 10.1177/2050640616663439.

26. Farmer $\mathrm{AD}$, Gallagher J, Bruckner-Holt C, Aziz Q. Narcotic bowel syndrome. Lancet Gastroenterol Hepatol. 2017;2(5):361-368. doi: 10.1016/S2468-1253(16)30217-5.

27. Omer A, Quigley EMM. An update on prucalopride in the treatment of chronic constipation. Therap Adv Gastroenterol. 2017;10(11):877-887. doi: 10.1177/1756283X17734809.

28. Nee J, Zakari M, Sugarman MA, Whelan J, Hirsch W, Sultan S, et al. Efficacy of Treatments for Opioid-Induced Constipation: Systematic Review and Meta-analysis. Clin Gastroenterol Hepatol. 2018;16(10):1569-1584.e2. doi: 10.1016/j.cgh.2018.01.021. 